\title{
Photomechanical effect on Type I collagen using pulsed diode laser
}

\author{
Gu-In Jung ${ }^{\mathrm{a}, 1}$, Ji-Sun Kim ${ }^{\mathrm{a}, 1}$, Tae-Hee Lee ${ }^{\mathrm{a}}$, Ju-Hyeon Choi ${ }^{\mathrm{a}}$, Han-Byeol Oh ${ }^{\mathrm{a}}$, A-Hee Kim ${ }^{\mathrm{a}}$, \\ Jun-Sik Kim ${ }^{\mathrm{a}}$, Jong-Rak Park ${ }^{\mathrm{b}}$, Soon-Cheol Chung ${ }^{\mathrm{a}}$, Dong-Il Yeom ${ }^{\mathrm{c}, \mathrm{d}}$, Hyung-Sik Kim ${ }^{\mathrm{a}}$ and \\ Jae-Hoon Jun ${ }^{\text {a,* }}$ \\ ${ }^{\mathrm{a}}$ Department of Biomedical Engineering, BK21+ Research Institute of Biomedical Engineering, \\ College of Biomedical and Health Science, Konkuk University, Chungju, Korea \\ ${ }^{\mathrm{b}}$ Department of Photonic Engineering, Chosun University, Gwangju, Korea \\ ${ }^{\mathrm{c}}$ Department of Physics, Ajou University, Suwon, Korea \\ ${ }^{\mathrm{d}}$ Department of Energy Systems Research, Ajou University, Suwon, Korea
}

\begin{abstract}
.
BACKGROUND: As the most abundant protein in human tissues, the use of collagen is essential in the fields of biological science and medicine.

OBJECTIVE: The aim of this study is to investigate the mechanical effect of pulsed laser irradiation on collagen tissue.

METHODS: With various laser parameters such as peak power, pulse width, and repetition rate, the induced stresses on samples were measured and analyzed. Monte Carlo simulation was performed to investigate the effect of laser parameters on the collagen sample.

RESULTS: The results indicated that the magnitude of mechanical stress could be controlled by various laser parameters.

CONCLUSIONS: This study can be used in biostimulation for therapy and mechanoreceptor stimulation for tactile application.
\end{abstract}

Keywords: Biostimulation, laser-tissue interaction, photomechanical effect, recoil pressure, thermoelastic effect

\section{Introduction}

For many decades, laser technology has developed in many fields such as industry, biology, and medicine because of the unique characteristic of the laser [1|2]. Unlike conventional light source which spread lights in all directions, laser light is collimated without divergence. Other beneficial characteristics are coherent and monochromatic [3]. In medical applications, the mostly used wavelength of the laser is in ultraviolet, visible, and infrared range [4]5]. Each wavelength of laser interacts differently with biological tissues [6-8].

When laser energy is irradiated on tissue, the photon penetrates into the tissue. The energy is distributed within the tissue depending on the tissue optical properties such as scattering coefficient, absorption coefficient, and refractive index. A portion of this energy can be absorbed by the tissue and

\footnotetext{
${ }^{1}$ These authors contributed equally to this work.

${ }^{*}$ Corresponding author: Jae-Hoon Jun, Department of Biomedical Engineering, College of Biomedical and Health Science, Konkuk University, 268 Chungwondaero, Chungju, Chungbuk 380-701, Korea. Tel.: +82 43840 3799; E-mail: jjun81@ kku.ac.kr.
} 
converted into thermal energy which makes the laser energy as a heat source. This laser-induced heat source initiates a non-equilibrium process of heat transfer manifesting itself by a temperature rise in tissue. The combined mechanisms of conduction, convection and emissive radiation distribute the thermal energy in the tissue resulting in temperature distribution in the tissue. The temperature distribution depends on the thermal properties of the tissue [9].

In laser-tissue interaction, excessive heat deposition by laser can cause thermal injury to the tissue. Also laser can induce stress in tissues, and such mechanisms of interaction are described as photomechanical effect. Under stress, the tissue can be deformed, either reversibly or irreversibly. If the stress is sufficient, the tissue can break. Transient stress waves can be generated by several mechanisms. Sudden thermoelastic expansion of tissue, caused by rapid heating by a pulse laser, can cause recoil momentum inside tissues [10-12].

The purpose of this study is to investigate the mechanical effect caused by pulsed laser irradiation on biological tissue. Various laser parameters (e.g., peak power, pulse width, and repetition rate) were applied to measure the induced stress on collagen samples. The manufactured Type I collagen was used as tissue phantom because collagen is the most abundant protein in human tissues. The results can be used in low-power biostimulation for therapy and mechanoreceptor stimulation for tactile application.

\section{Materials and methods}

\subsection{Tissue phantom}

Since collagen is the most abundant protein in human tissues such as skin, tendon, bone, and blood vessels, the understanding of collagen is essential in the fields of biological science and medicine [13]. As phantom tissue model, we used the film membrane, Type I collagen of fish origin sample (NeuSkin$\mathrm{F}^{\circledR}$, Medira Ltd., United Kingdom). The dimension of the collagen sample is $25 \times 25 \mathrm{~mm}^{2}$. Skin is the first boundary for laser energy to be applied to biological tissues and its thickness varies considerably according to the race, age, and region of the body surface [14]. Thus, various numbers of the sample (5, $10,15,20$, and 25$)$ were applied to change the sample thicknesses $(0.21 \sim 0.91 \mathrm{~mm})$.

\subsection{Experimental procedure and laser parameters}

Figure 1 shows the experimental setup to detect the mechanical impact of laser on the collagen sample. The fiber-coupled diode laser system (Pearl ${ }^{\mathrm{TM}}$ TKS-B, nLight Photonics Corp., Santa Clara, CA; wavelength $=809 \mathrm{~nm}$, fiber core diameter $=400 \mathrm{um}$, and numerical aperture $=0.22$ ) has a function to change laser parameters (e.g., pulse width $=10 \mu \mathrm{s} \sim \mathrm{CW}$, repetition rate $<12.5 \mathrm{KHz}$, and peak power $=35 \mathrm{~W}$ ) precisely with computer-controlled parameter selection.

Laser beam passing through collimating lenses is divided by beam splitters (BS1 and BS2) into collagen sample, pulse detector, and energy meter to detect pulse width and laser energy, and to focus on collagen sample by a lens (L3). The collagen sample is attached on polyvinylidene fluoride (PVDF) transducer which can measure the mechanical stress induced by pulsed laser energy. Spot diameter on the sample is set to $3.5 \mathrm{~mm}$ by lenses and 3-axis translation stage. Pulse width, pulse energy, and beam diameter can be measured with pyroelectric energy detector (818E-05-12-L, Newport, Irvine, CA), energy meter (1918-R, Newport, Irvine, CA), and beam profiler (SP620U, Ophir Optronics Inc., Danvers, $\mathrm{MA})$, respectively.

Figure 2 shows laser parameters considered in this study and the example of PC controlled platform for laser parameter control. Peak power $(3.88 \sim 13.85 \mathrm{~W})$, pulse width $(20 \sim 100 \mu \mathrm{s})$, and repetition rate $(10 \mathrm{~Hz})$ were applied to the experiment with an episode time of 5 secs. 


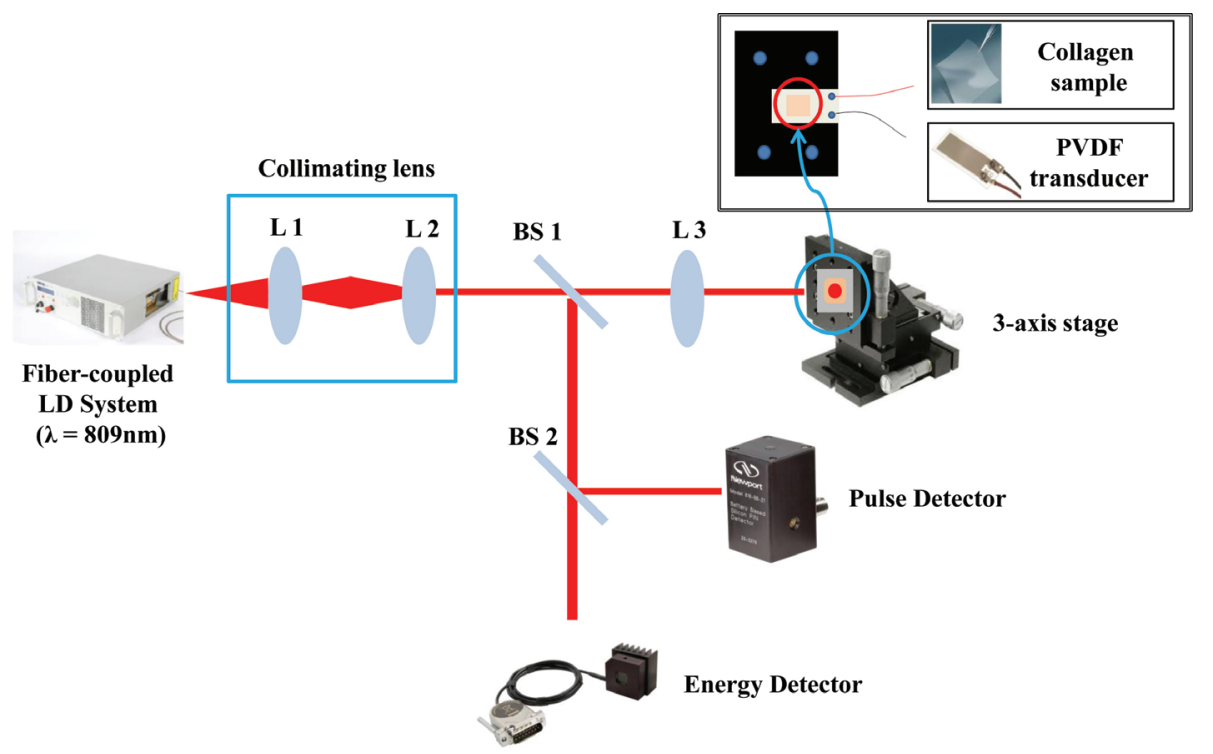

Fig. 1. Schematic diagram of the experimental setup to detect the photomechanical effect.

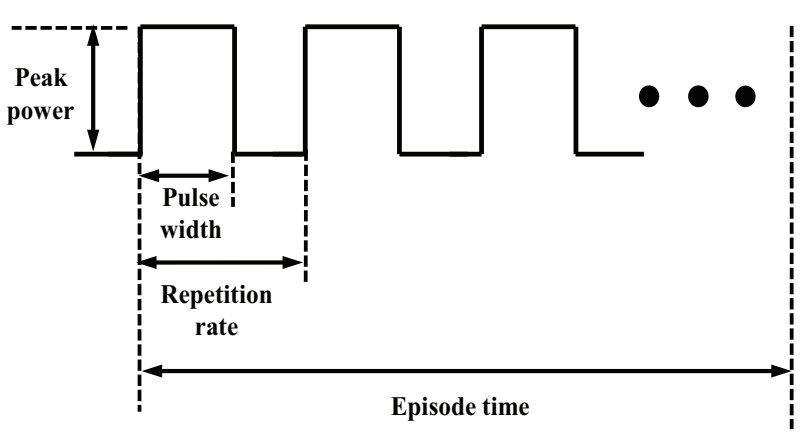

(a) Laser parameters

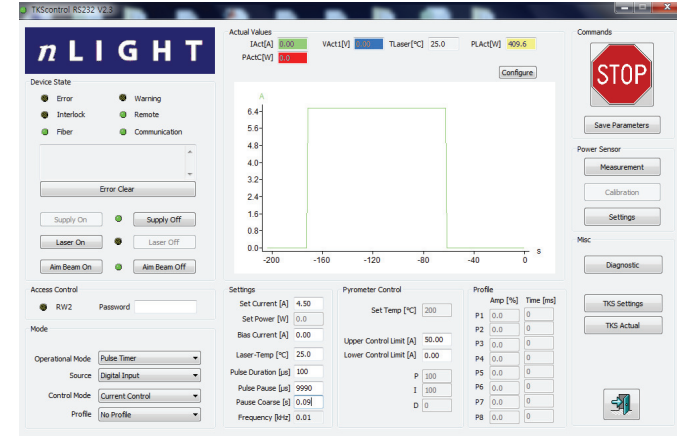

(b) Laser operating software

Fig. 2. Laser parameters and the laser operating software.

\subsection{Signal acquisition and processing}

A polyvinylidene fluoride (PVDF) is a polymer with pyroelectric and piezoelectric properties that make it suitable to measure mechanical stresses. The PVDF transducer (LDT1-028K, Measurement Specialties, Hampton, VA) was used to detect the photomechanical effect by laser irradiation. The film sensor is a flexible component comprising a $28 \mu \mathrm{m}$ thick piezoelectric PVDF polymer film with screenprinted Ag-ink electrodes, laminated to a $0.125 \mathrm{~mm}$ polyester substrate, and fitted with two crimped contacts. As the piezo film is displaced from the mechanical neutral axis, bending creates very high strain within the piezopolymer and, therefore high voltages are generated. The sensitivity of the film sensor was $13 \mathrm{mV} / \mathrm{N}$. The output signals were magnified by the amplifier (Piezo Film Lab Amplifier, Measurement Specialties, Hampton, VA) to $40 \mathrm{~dB}$, converted to digital signals with the data acquisition board (NI USB-6361, National Instruments, Austin, Texas), and saved using LabVIEW program (NI LabVIEW 2012, National Instruments, Austin, Texas). 


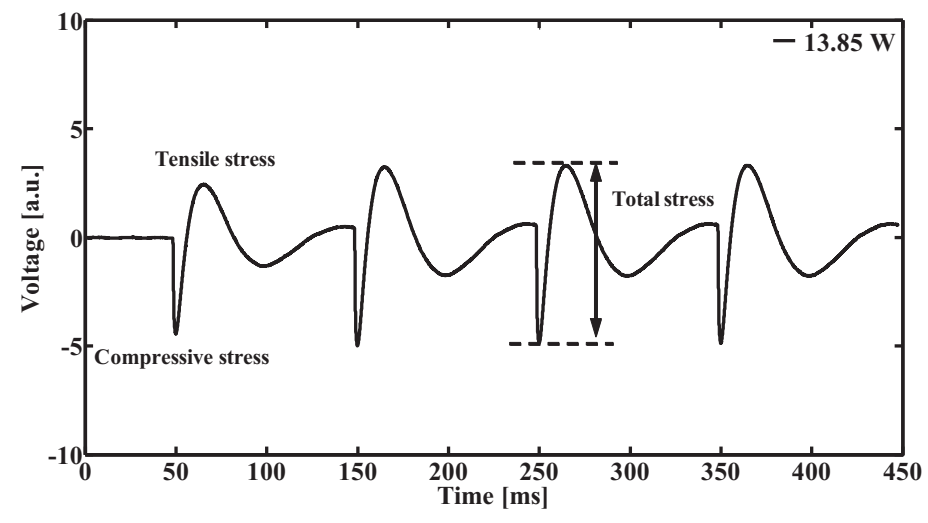

Fig. 3. Pressure signals obtained with PVDF transducer.

\subsection{Monte Carlo simulation}

Monte Carlo simulation was performed to investigate the effect of laser irradiation on the collagen samples with TracePro (Lambda Research Corp., Littleton, MA) program. Input laser parameters for the simulation were laser power $(3.88 \mathrm{~W} \sim 13.85 \mathrm{~W})$, pulse width $(20 \mu \mathrm{s} \sim 100 \mu \mathrm{s})$, beam size $(3.5 \mathrm{~mm})$, wavelength $(809 \mathrm{~nm})$, and the number of photon $(3,000,000)$. The optical parameters of collagen sample were refractive index $(n=1.4)$, absorption coefficient $\left(\mu_{a}=11.1 \mathrm{~mm}^{-1}\right)$, scattering coefficient $\left(\mu_{s}=\right.$ $\left.11.1 \mathrm{~mm}^{-1}\right)$, and anisotropic factor $(g=0.8)$ [15-17]. With this simulation technique, we can obtain the light energy and temperature distributions at a certain depth of the collagen sample to compare with experimental results.

\section{Results and discussion}

\subsection{PVDF transducer and temporal profile}

Figure 3 shows the pressure signals from PVDF transducer by the laser irradiation on the collagen sample. The applied laser parameters were pulse width $(100 \mu \mathrm{s})$, peak power $(13.85 \mathrm{~W})$, and repetition rate $(10 \mathrm{~Hz})$. Temporally measured output signals are represented in terms of voltage [V]. Bipolar waveforms imply that the compressive and tensile stresses were consistently produced. The front negative peaks were created by the compressive-stress wave arriving first and the subsequent positive peaks by the tensile-stress wave following after the compressive-stress wave. The results indicated that the pulsed laser irradiation could cause mechanical stresses in the bipolar pattern by induced thermo-elastic effect.

\subsection{Collagen thickness effect}

Figure 4 shows the total stress under the condition with pulse width $(20 \sim 100 \mu \mathrm{s})$, thickness of samples $(0.21 \sim 0.91 \mathrm{~mm})$, and laser power $(3.88 \sim 13.85 \mathrm{~W})$, induced by thermoelastic effect. With the same power, the total stress is decreased as the thickness of sample is increased. This result implies that the laser energy and stress intensity are reduced at the deeper position of the sample. Similar patterns are observed at different pulse width. 

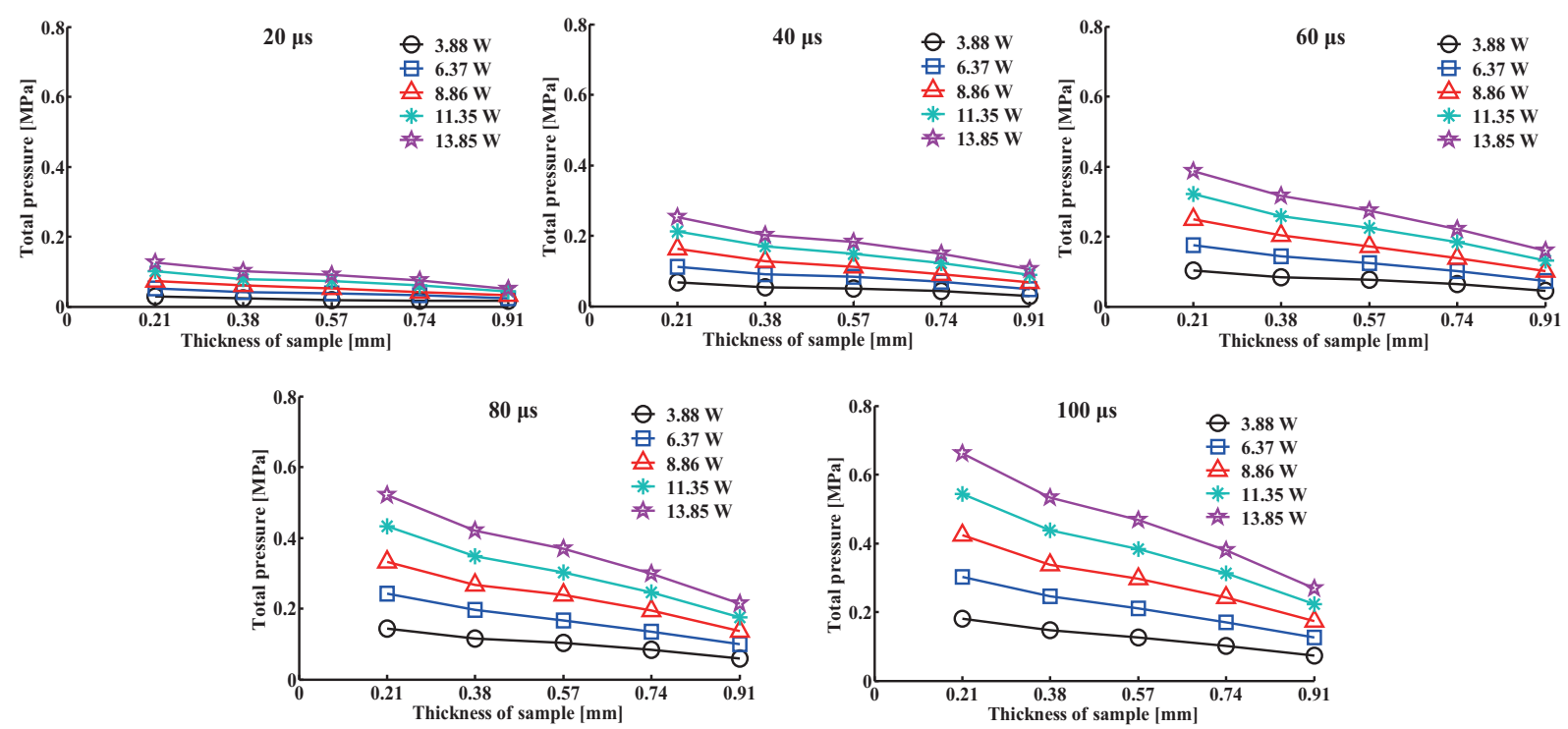

Fig. 4. Total stress changes caused by laser power and the thickness of sample.
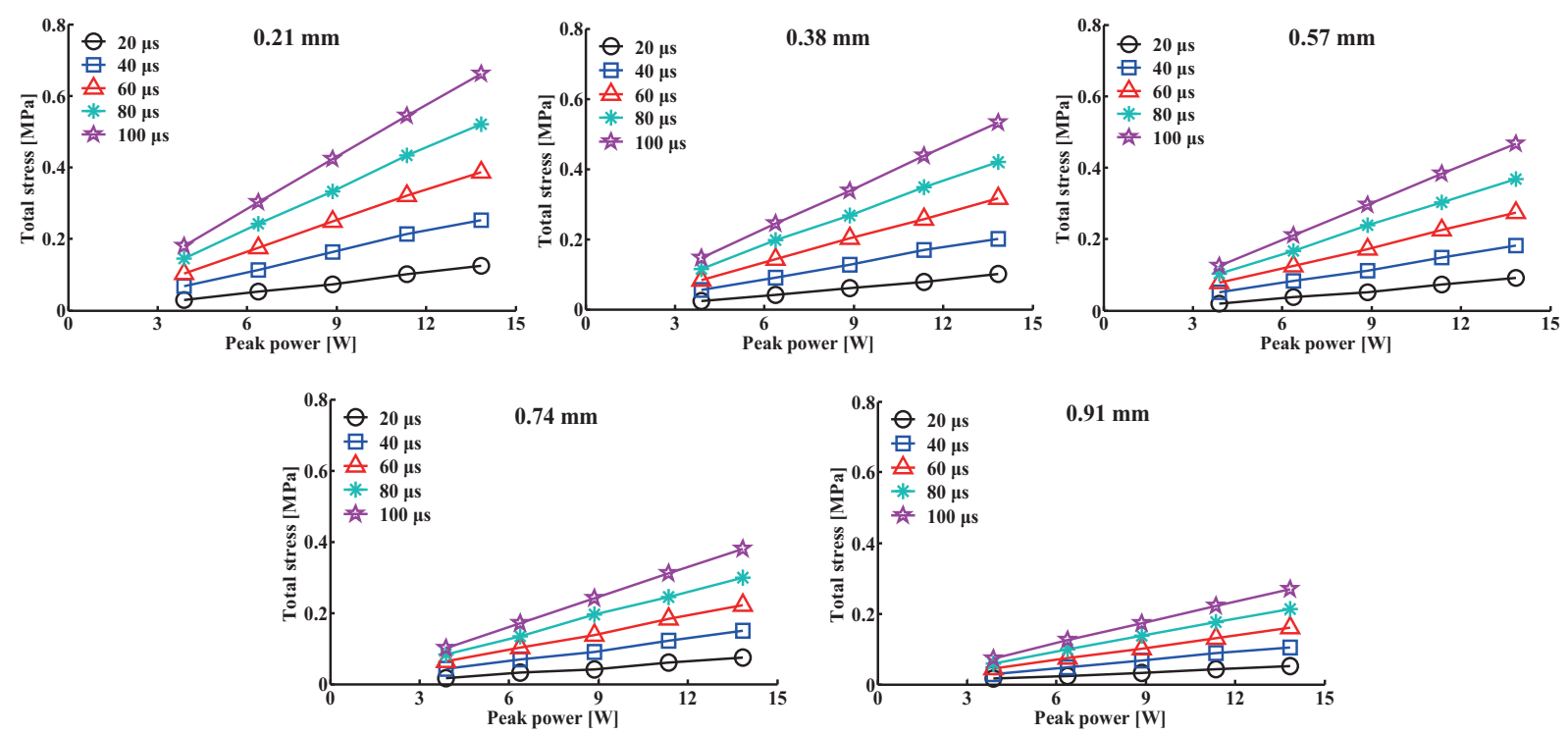

Fig. 5. Total stress changes caused by laser power and pulse width.

\subsection{Pulse width effect}

Figure 5 shows the change of total stress due to pulse width and laser power. As the pulse width and laser power are increased, the detected total stress is increased. Under the same power, the energy per pulse is increased as the pulse width is increased. The result implies that the mechanical stress depends on the induced energy per pulse.

Figure 6 shows the overall experimental results. The magnitude of the induced stress is increased linearly as laser power and pulse width are increased, while the induced stress is decreased as the thickness 


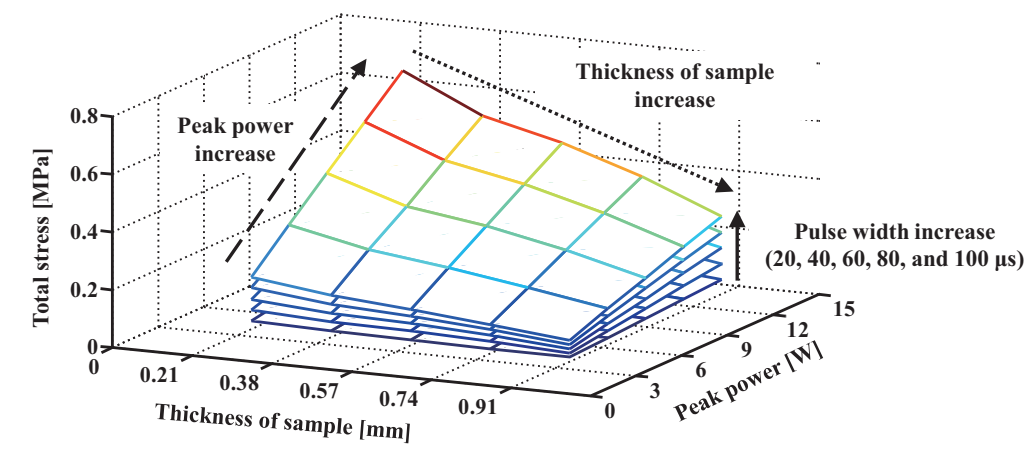

Fig. 6. Measured total stress varied with laser parameters.
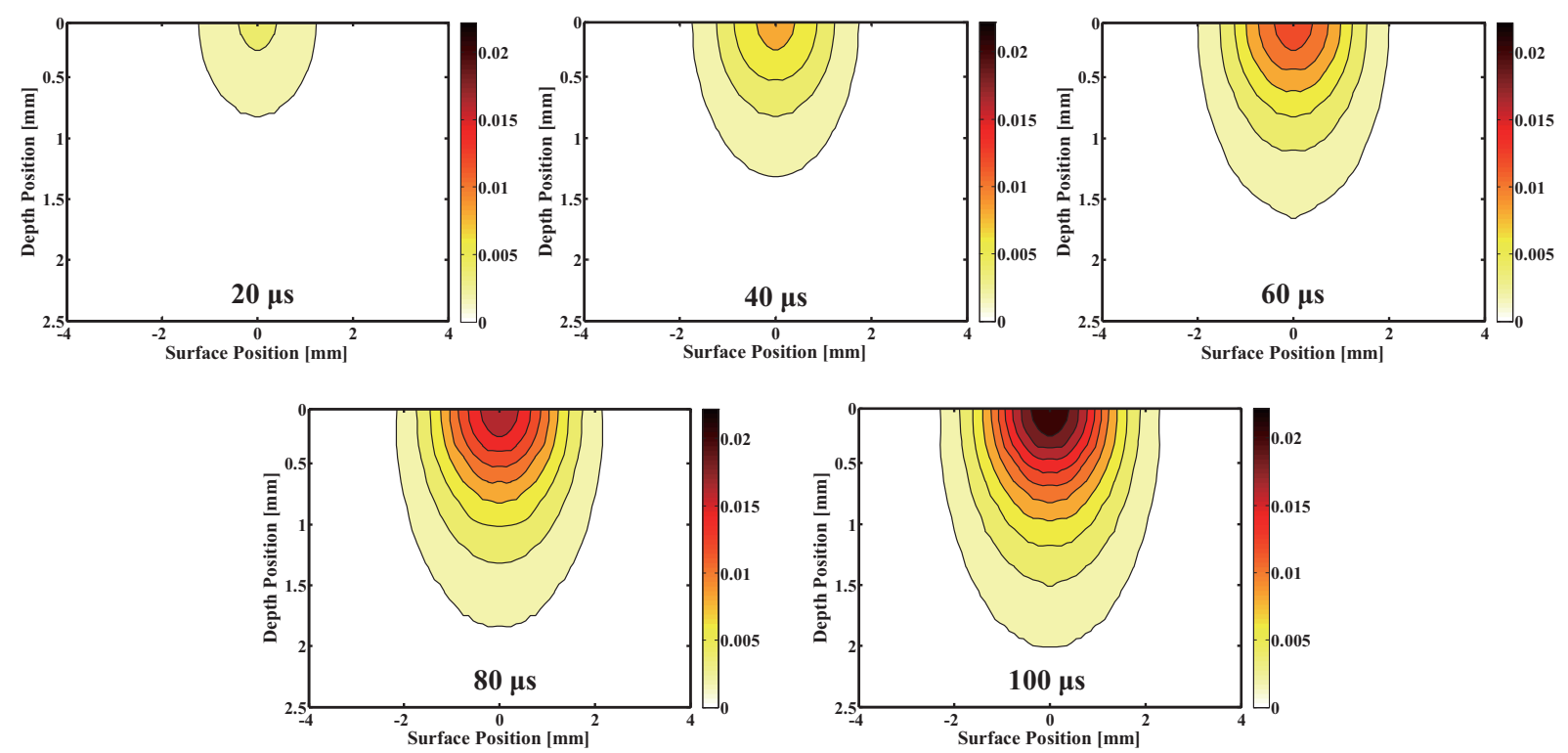

Fig. 7. Temperature distribution changes in the collagen sample (peak power $=13.85 \mathrm{~W})$.

of sample is increased. These results imply that the degree of stress can be controlled by various laser parameters.

\subsection{Monte Carlo simulation results}

Figure 7 shows the theoretical results by performing a Monte Carlo simulation. The results show the temperature changes due to the sample depth with laser parameters of wavelength $(809 \mathrm{~nm})$, laser power $(13.85 \mathrm{~W})$, and pulse width $(20 \mu \mathrm{s} \sim 100 \mu \mathrm{s})$. At center of the laser beam, the temperature rise shows the maximum value and the temperature are decreased as the depth is increased. With the same power, the maximum temperature rise $\left(\Delta T_{\max }\right)$ is increased as the pulse width is increased. The temperature rise is in the range of $0.004^{\circ} \mathrm{C} \sim 0.022^{\circ} \mathrm{C}$.

The simulation results show that the more energy and mechanical stress are transferred into the sample as the pulse width is increased. The induced stress by thermoelastic effect is increased as the temperature change is increased. The theoretical results are correlated well with the experimental results. 


\section{Conclusions}

The laser-induced mechanical effect was investigated with collagen samples by changing laser parameters. Monte Carlo simulation was performed to be compared with experimental results. The pulsed laser energy can be converted to mechanical stress in collagen by thermoelastic effect which results in physical displacement of the sample. The results imply that the degree of stress can be controlled with various laser parameters. In future study, the effect of laser parameters on mechanoreceptors in skin will be investigated to apply the results for the study of ion channel activity, therapeutic biostimulation, and non-contact tactile stimulation without tissue damage.

\section{Acknowledgements}

This research was supported by the Pioneer Research Center Program through the National Research Foundation of Korea funded by the Ministry of Science, ICT \& Future planning (No. 2011-0027920).

\section{References}

[1] Abuarra A, Abuarra B, Abur BS, Singh GK, AlSadi Z, Mahmood TLIR, et al. The effects of different laser doses on skin. Int. J. Phys. Sci. 2012; 7(3): 400-407.

[2] Dubey AK, Yadava V. Laser beam machining - a review. Int. J. Mach. Tools Manuf. 2008; 48(6): 609-628.

[3] Caroll LS, Humphreys TR. Laser-tissue interactions. Clin. Dermatol. 2006; 24(1): 2-7.

[4] Lanigan SW. Lasers in dermatology. Medicine. 2004; 32(12): 21-23.

[5] Stritzker J, Kirscher L, Scadeng M, Deliolanis NC, Morscher S, Symvoulidis P, et al. Vaccinia virus-mediated melanin production allows MR and optoacoustic deep tissue imaging and laser-induced thermotherapy of cancer. PNAS. 2013; 110(9): 3316-3320.

[6] Omar KM. Laser effects in skin melanin. Mod. App. Sci. 2009; 3(1): 57-62.

[7] Wang M, Ning Y, Zou H, Chen S, Bai Y, Wang A, et al. Effect of Nd: YAG laser-nitriding-treated titanium nitride surface over Ti6A14V substrate on the activity of MC3T3-E1 cells. Bio-Med. Mater. Eng. 2014; 24(1): 643-649.

[8] Chen N, Chen M, Liu S, Guo Q, Chen Z, Wnag T. Change in refractive index of muscle tissue during laser-induced interstitial thermotherapy. Bio-Med. Mater. Eng. 2014; 24(1): 807-813.

[9] Enderle JD, Bronzino JD. Introduction to biomedical engineering, Academic Press; 2012.

[10] Jacques SL. Laser-tissue interactions. Photochemical, photothermal, and photomechanical. Sug. Clin. North Am. 1992; 72: 531-558.

[11] Boulnois JL. Photophysical processes in recent medical laser developments: a review. Laser Med. Sci. 1986; 1(1): 47-66.

[12] Paltauf G, Dyer PE. Photomechanical processes and effects in ablation. Chem. Rev. 2003; 103(2): 487-518.

[13] Jun JH, Harris JL, Humphrey JD, Rastegar S. Effect of thermal damage and biaxial loading on the optical properties of a collagenous tissue. J. Biomech. Eng. 2003; 125(4): 540-548.

[14] Lee Y, Hwang K. Skin thickness of Korean adults. Surg. Radiol. Anat. 2002; 24(3-4): 183-189.

[15] Bashkatov AN, Genina EA, Kochubey VI, Tuchin VV. Optical properties of human skin, subcutaneous and mucous tissues in the wavelength range from 400 to 2000 nm. J. Phys. D: Appl. Phys. 2005; 38(15): 2543-2555.

[16] Salomatina E, Jiang B, Novak J, Yaroslavsky AN. Optical properties of normal and cancerous human skin in the visible and near-infrared spectral range. J. Biomed. Opt. 2006; 11(6): 064026-064026.

[17] Zonios G, Dimou A. Light scattering spectroscopy of human skin in vivo. Opt. Express. 2009; 17(3): 1256-1267. 